\title{
Predicting the Parameters of Vortex Bladeless Wind Turbine Using Deep Learning Method of Long Short-Term Memory
}

\author{
Mahsa Dehghan Manshadi ${ }^{1}$, Majid Ghassemi ${ }^{1}$, Seyed Milad Mousavi ${ }^{1}$ (D), Amir H. Mosavi ${ }^{2, *}$ (D) \\ and Levente Kovacs ${ }^{3,4,5}$ (D) \\ 1 Department of Mechanical Engineering, K.N. Toosi University of Technology, Tehran 1999143344, Iran; \\ mahsadehghan@email.kntu.ac.ir (M.D.M.); ghasemi@kntu.ac.ir (M.G.); \\ Miladmousavi@email.kntu.ac.ir (S.M.M.) \\ 2 Institute of Software Design and Development, Obuda University, 1034 Budapest, Hungary \\ 3 Biomatics Institute, John von Neumann Faculty of Informatics, Obuda University, 1034 Budapest, Hungary; \\ kovacs@uni-obuda.hu \\ 4 ELKH SZTAKI Institute, P.O. Box 63, 1518 Budapest, Hungary \\ 5 Physiological Controls Research Center, University Research and Innovation Center, Obuda University, \\ 1034 Budapest, Hungary \\ * Correspondence: amir.mosavi@uni-obuda.hu
}

Citation: Dehghan Manshadi, M.; Ghassemi, M.; Mousavi, S.M.; Mosavi, A.H.; Kovacs, L. Predicting the Parameters of Vortex Bladeless Wind Turbine Using Deep Learning Method of Long Short-Term Memory. Energies 2021, 14, 4867. https://doi.org/ $10.3390 /$ en14164867

Academic Editor: Oscar Barambones

Received: 7 June 2021

Accepted: 6 August 2021

Published: 9 August 2021

Publisher's Note: MDPI stays neutral with regard to jurisdictional claims in published maps and institutional affiliations.

Copyright: (C) 2021 by the authors Licensee MDPI, Basel, Switzerland. This article is an open access article distributed under the terms and conditions of the Creative Commons Attribution (CC BY) license (https:/ / creativecommons.org/licenses/by/ $4.0 /)$.

\begin{abstract}
From conventional turbines to cutting-edge bladeless turbines, energy harvesting from wind has been well explored by researchers for more than a century. The vortex bladeless wind turbine (VBT) is considered an advanced design that alternatively harvests energy from oscillation. This research investigates enhancing the output electrical power of VBT through simulation of the fluid-solid interactions (FSI), leading to a comprehensive dataset for predicting procedure and optimal design. Hence, the long short-term memory (LSTM) method, due to its time-series prediction accuracy, is proposed to model the power of VBT from the collected data. To find the relationship between the parameters and the variables used in this research, a correlation matrix is further presented. According to the value of 0.3 for the root mean square error (RMSE), a comparative analysis between the simulation results and their predictions indicates that the LSTM method is suitable for modeling. Furthermore, the LSTM method has significantly reduced the computation time so that the prediction time of desired values has been reduced from an average of two and a half hours to two minutes. In addition, one of the most important achievements of this study is to suggest a mathematical relation of output power, which helps to extend it in different sizes of VBT with a high range of parameter variations.
\end{abstract}

Keywords: wind turbine; computational fluid dynamics; deep learning; long short-term memory; energy; artificial intelligence; renewable energy; machine learning; data science; energy conversion

\section{Introduction}

These days, global warming due to the environmental pollution caused by fuels is one of the crucial existing issues of developing countries [1]. Hence, using renewable energy devices as an energy converter has become common recently. The prevalent renewable energy sources are wind power, ocean wave power, biomass power, and photovoltaic power [2]. One of the most accessible sources of sustainable energies among introduced sources is wind power. Several projects have been performed on a large scale to utilize these sources all over the world [3]. Although conventional wind turbines are so costly and have a bad environmental effect [4], researchers are finding an effective way to design a type of turbine that is cheap and economical [5]. This important issue inspired engineers to excogitate a vortex bladeless wind turbine (VBT) [6]. Another crucial concern in renewable energy systems is to predict the produced power of a system due to the farm location without spending money. System production and installation need investments, and one 
of the important aspects of this study is to forecast the produced power of VBT with related parameters.

VBT is one of the newest bladeless turbines proposed by researchers, which is the beginning of a great revolution in this industry. This type of turbine does not have the problems of the previous generation turbines, so to optimize VBT [7], many researchers around the world have started experimental and research studies. Antony and Boucher [8] have studied the effective parameters on the output power of the bladeless turbine. They showed that for higher and lower wind speeds, the turbine vibration parameters are constant. The results of the numerical solution of the equations demonstrate that high amplitudes occur only in vibration resonance. Sassi et al. [9] have utilized the discrete element method with the immersed boundary method to solve Navier-Stokes equations. By analyzing different vibrational parameters and solving differential equations by the Runge-Kutta method, they have concluded that the efficiency values are between $20 \%$ and $30 \%$ in the lock-in range. Gautam et al. [10] performed numerical analyses on the electrical part and the production of turbine power without blades using commercial software. They found that the design of an electromechanical conversion system was essential to achieve the maximum electrical output for a given vibration frequency. They concluded that the different field settings with respect to the coil are crucial for the use of electrical power. Moradi Gharghani et al. [11] have studied the effect of a dimensionless Reynolds number on body oscillation. They concluded that there is a unique Reynolds number for which the frequency of the vortices is equal to the maximum frequency of the turbine oscillation and the maximum power happens. Among these studies, the relationship between the multi-physic parameters, i.e., wind flow velocity and oscillation amplitude and frequency, wind flow velocity and output power, drag force and oscillation amplitude and frequency, has not yet been clarified, so it is not evident which one is significant in these kinds of simulations.

Due to the attractiveness of this issue for investors in the field of renewable energy around the world [12] and the significant progress of this type of turbine by experts in this field, it is necessary to conduct more extensive studies on various aspects of this project, to expand the use of these turbines around the world. One of the most important areas that can be mentioned for the optimization of VBT is the maximization of production power. Therefore, in order to use the effective parameters on the output power optimization, which has not been studied so far, it is necessary to form a new study. Accordingly, this study aims to estimate the power output and further enhances the parameters related to efficiency, which are the essential challenges in the VBT optimal design. Investigation of the effects of fluid and solid parameters in the analysis of the fluid-solid interactions (FSI) on the output power of VBT can be performed by solving the coupled equations by computational fluid dynamics (CFD) commercial software. In order to predict the optimal amount of generated power, it is necessary to use new methods that have recently been introduced in the field of predicting the amount of electricity generated by energy systems [13], especially wind turbines. In these fields, several studies have been performed, especially utilizing the output power from wind energy systems. Some researchers studied an advanced constructed data-driven model for predicting the output power by using a neural network algorithm [14]. The output power of VBT varies with different parameters, i.e., wind flow velocity, rod deflection (oscillation amplitude), and exerted drag force [15]. Therefore, it is crucial to design a suitable algorithm to develop the prediction model. The latest modified method of predicting this type of system is the use of artificial intelligence (AI) [16]. One of the most well-known methods is LSTM [17], and many studies have used this method for optimizing the prediction of output power produced from wind turbines in different areas with different geographical properties. Yang et al. [18] conducted studies on wind power prediction that used the LSTM method for predicting the desired data. They compared the predicted power tables with the available data on the actual turbine power output measured in the wind farm. Wu et al. [19] studied the effective parameters for optimizing the output power of wind turbines and compared the results 
with the experimental data available from a wind farm in China. They concluded that the LSTM method can accurately detect values, is very accurate and fast, and is more efficient at predicting values than other existing methods. Meka et al. [20] analyzed the information of turbines of a power plant, and by drawing diagrams that predict the parameters by the LSTM method, they concluded that it is one of the best and most efficient methods in this field. According to the latest studies in this field, it can be concluded that the LSTM method is one of the best methods for VBT analysis, which is one type of wind turbine. Related to the importance of the power prediction in VBT, this study used an AI algorithm to make a prediction. The innovation in this research can be justified by the fact that the use of this method in wind turbines without blades has not been mentioned in an article. In this novel study, the proposed method is to combine the two-way FSI, which is achieved by the CFD method based on the finite element method. The numerical datasets were used in the LSTM algorithm to estimate electrical power using input variables. The novelties can be summarized as follows. (a) the multi-physics numerical analysis in the form of the fluid-solid interactions is proposed for the first time by analyzing vortex bladeless wind turbines in a computational fluid dynamic space, which completely demonstrates the fluid parameters of the airflow and can perfectly combine with the most applicable algorithm of a deep neural network. (b) the long-short-term memory method is reasonably utilized to predict output electrical power for the first time based on the numerical simulations, which can predict the wind power of a vortex bladeless wind turbine. Furthermore, by suggesting a mathematical relation of generated power, many methods can be implemented to help the bladeless wind turbine industry to predict output power with less time and cost.

\section{Materials and Methods}

The VBT is a flexible cylindrical structure that oscillates in a fluid flow [21]. The special type of the VBT $1 \mathrm{~m}$ high was studied in this research [7]. Not only does this special design lack any blades for a rotational movement, but it also has a mast part for oscillating in any direction [22,23]. The vortex shedding phenomenon exerts lift force, which causes the structure to fluctuate crosswise. Using the effect of vortex-induced vibration (VIV) phenomenon in an energy harvesting procedure helps to convert fluid energies to the output electrical power by an aero-generator. The aero-generator system contains a group of moving magnets on a fixed coil that uses the Faraday law of electromagnetic induction [24]. Figure 1 presents the structure of VBT.

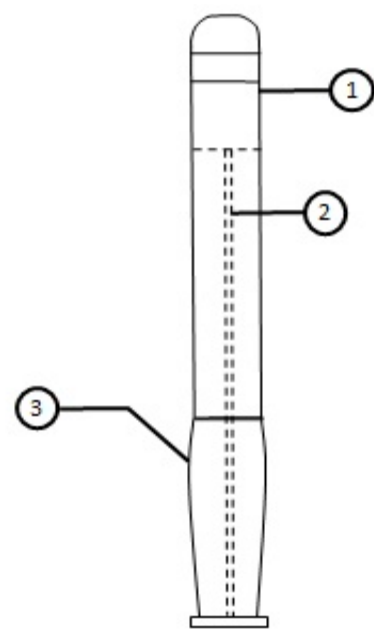

Figure 1. A schematic view of an standard vortex bladeless wind turbine.

As shown in Figure 1, the VBT structure has three main parts that are assembled together according to [7]. (a) Cylindrical mass made of glass fiber (Part 1). (b) Flexible rod made of reinforced carbon fiber that is fixed to part1 (Part 2). (c) Special cover that anchors the carbon fiber rod to the foundation (Part 3). The purpose of the lightweight cylindrical 
mass that is connected to the flexible rod is to harvest energy by converting the mechanical energy to an electrical form of energy.

\subsection{Governing Equations}

The governing equation is based on assumptions that the airflow is two-dimensional, steady, and incompressible, with constant properties considered at $20^{\circ} \mathrm{C}$ [25]. The twodimensional continuity equation of fluid is shown as Equation (1) according to [26].

$$
\frac{\partial u}{\partial x}+\frac{\partial v}{\partial y}=0
$$

where $\mathrm{u}$ and $v$ are the wind velocities in $x$ and $y$ directions. In this research, the $\mathrm{z}$-direction velocity $(w)$ was neglected because the VBT does not have any vibration in this direction. The momentum equations in the $\mathrm{x}$ and $\mathrm{y}$ directions are presented as Equations (2) and (3), respectively, as stated in [26].

$$
\begin{aligned}
& \frac{\partial \mathrm{u}}{\partial t}+\mathrm{u} \frac{\partial \mathrm{u}}{\partial x}+v \frac{\partial \mathrm{u}}{\partial y}=-\frac{1}{\rho} \frac{\partial p}{\partial x}+v\left(\frac{\partial^{2} \mathrm{u}}{\partial x^{2}}+\frac{\partial^{2} \mathrm{u}}{\partial y^{2}}\right) \\
& \frac{\partial v}{\partial t}+u \frac{\partial v}{\partial x}+v \frac{\partial v}{\partial y}=-\frac{1}{\rho} \frac{\partial p}{\partial y}+v\left(\frac{\partial^{2} v}{\partial x^{2}}+\frac{\partial^{2} v}{\partial y^{2}}\right)
\end{aligned}
$$

\subsection{Computational Domain and Boundary Conditions}

In recent research, the FSI analysis is performed by using the transient wind flow as the input. Inlet velocity is considered to have two-dimensional variables, and the pressure is assumed constant in the inlet and outlet. Based on these assumptions, the viscosity of the airflow varies with Reynold's number, which, in turn, is a function of different VBT diameters and wind velocity. Related to the input wind velocity, the maximum Reynolds number is $1.1 \times 105$. By considering this maximum value, the flow is assumed to be laminar before having interaction with VBT. When interactions between airflow and VBT happen, the vortex shedding street will change the flow regime to turbulent. The domain walls are constrained as a no-slip boundary condition, but the fluid-structure interfaces are assumed to slip walls. The study boundary conditions and dimensionless parameters are presented in Figure 2. The width and the length of the domain are $\mathrm{W}$ and $\mathrm{H}$. The upstream distance of the computational domain is designated by $\mathrm{W} 1$, while the downstream distance is designated by W2.

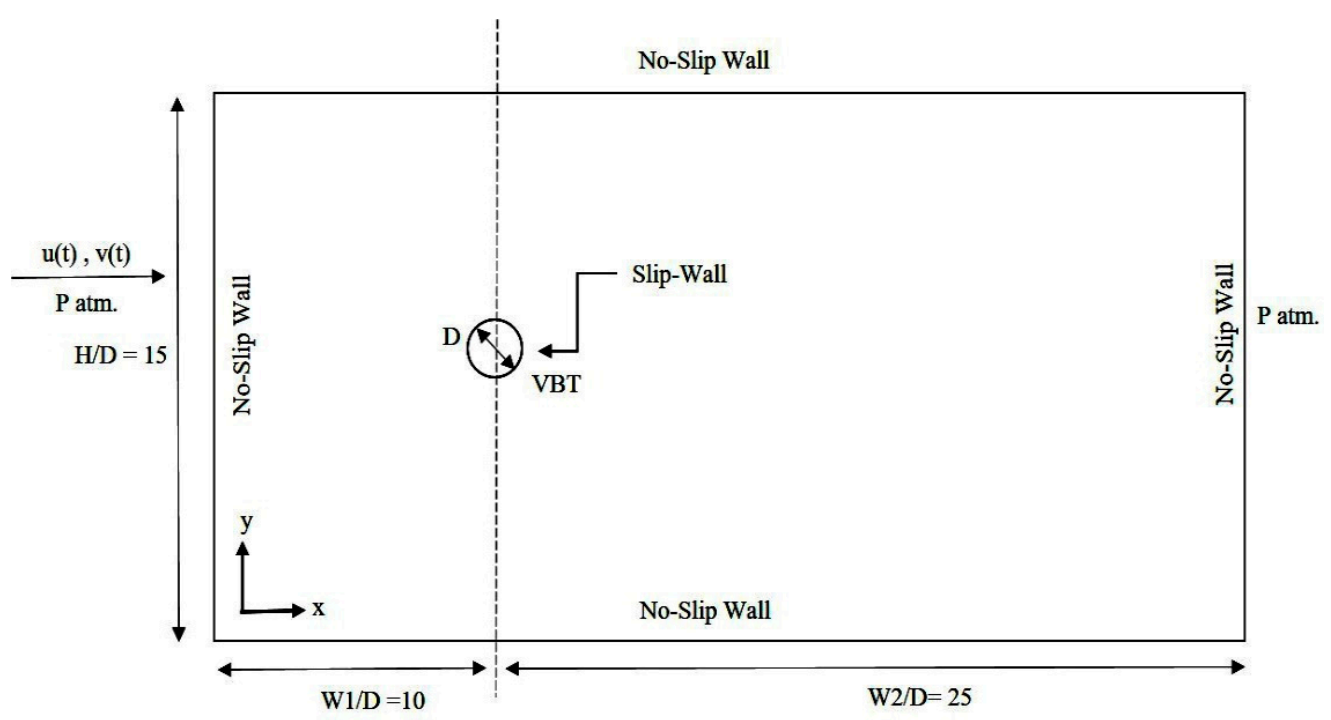

Figure 2. The schematic view of dimensionless parameters and boundary conditions. 


\subsection{Mesh Generation and Grid Independency}

Mesh generation analysis essentially acquired the amount of sufficient mesh for the purpose of this study.

Moreover, three different mesh numbers were generated to simulate a VBT, which is presented in Table 1.

Table 1. The information about mesh generation.

\begin{tabular}{cccc}
\hline Minimum Grid Size (m) & Simulation Time & Number of Elements & Accuracy of Oscillation Amplitude \\
\hline 0.01 & $34 \min 16 \mathrm{~s}$ & 19,921 & $85 \%$ \\
\hline 0.005 & $2 \mathrm{~h} 34 \min 7 \mathrm{~s}$ & 55,848 & $93 \%$ \\
\hline 0.001 & $5 \mathrm{~h} 54 \min 10 \mathrm{~s}$ & $2,244,300$ & $94 \%$ \\
\hline
\end{tabular}

As presented in Table 1, the grid number is increased in the computational domain to increase the accuracy of the numerical simulation. The criterion of selecting a minimum grid size results in a highly accurate answer due to the least simulation time. Three different triangular type mesh sizes are utilized, which are given in Table 1. Based on the least simulation time criterion, the $0.05 \mathrm{~m}$ grid size with 55,848 number of elements and about $2 \mathrm{~h}$ and $34 \mathrm{~min}$ run time is selected [27]. One of the selected parameters, in order to evaluate the accuracy, is the oscillation amplitude. Because the accuracy of the selected grid size is as high as the minimum one, 55,848 elements were selected because of the low calculation time and cost.

\subsection{Energy Conversion between Fluid and Structure}

Energy conversion in wind turbines has the general meaning of converting the wind mechanical energy to output electrical energy. In VBT systems, the wind mechanical energy converts to output electrical energy by using the body vibration. Figure 3 depicts a schematic of the simplified VBT model, which is considered as a simple springdamper model.

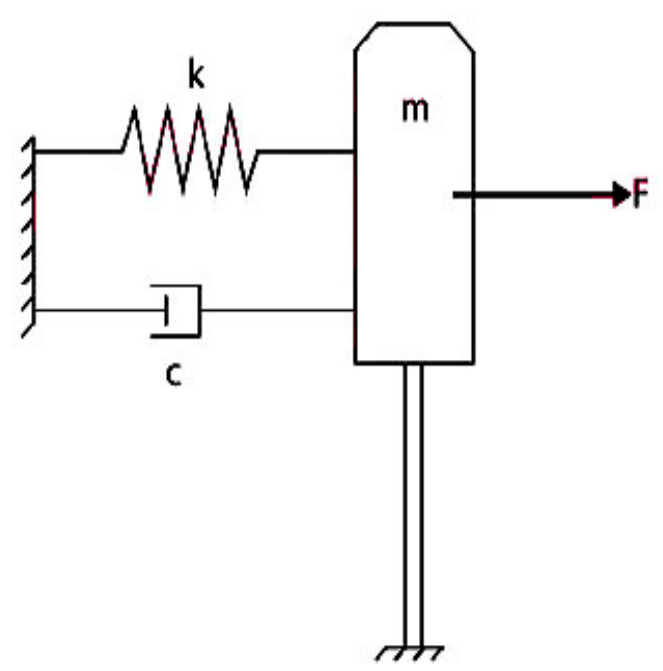

Figure 3. The simplified vibrational system of VBT.

Where " $m$ ", " $k$ ", " $c$ ", and $F$ are the mass of the vibrational parts of VBT, the structural stiffness, and the wind force, which causes the VBT to vibrate, respectively. The general structure vibration equation presented in the y-direction is as follows, according to [28].

$$
m \ddot{y}+k y+c \dot{y}=F_{\text {fluid }}(x \cdot y \cdot t)
$$


The direction of vibration of the VBT is perpendicular to the direction of the wind. The wind flow is presented in the $\mathrm{x}$-direction to simplify the equations. Respectively, another direction will be analyzed the same. The force exerted by the fluid is determined as follows, according to [29].

$$
F_{\text {fluid }}(x . y . t)=\frac{1}{2} \rho u^{2}(D l) C_{d}(x . y . t) \hat{i}+\frac{1}{2} \rho v^{2}(D l) C_{d}(x \cdot y \cdot t) \hat{j}
$$

Hence, the assumed inlet velocity variable with time and the interfaces of the VBT remain constant in the various directions due to the symmetric shape of the cylinder. In the equation, $\rho$ stands for the flow density and is constant, where $D$ represents the bigger VBT diameter. Furthermore, the VBT height is set as 1 . Due to the symmetrical shape of the cylinder, it can be proven that the VBT interfaces are the same in all directions. Therefore, the vibration is independent of the wind flow direction. $C_{d}(x, y, t)$ is the drag coefficient as is given by a harmonic equation.

This equation is considered a harmonic one, so we have the sine term inside it. $\varphi$ is the phase angle, and $\omega$ is angular velocity, which is larger than regular frequency $f$ by a factor of $2 \pi$, where $f$ is the flow frequency. By substituting Equation (4) in Equation (5), Equation (6) is obtained from [28].

$$
F_{\text {fluid }}(x . y . t)=\frac{1}{2} \rho u^{2}(D l) C_{d}(x . y) \sin (\omega t+\varphi) \hat{i}+\frac{1}{2} \rho v^{2}(D l) C_{d}(x . y) \sin (\omega t+\varphi) \hat{j}
$$

VIV is a phenomenon that occurs with coupling between vortex shedding and structural vibration. Figure 4 presents the schematic of the VBT, the two-way fluid-solid interactions, and the vortex shedding effect [29].

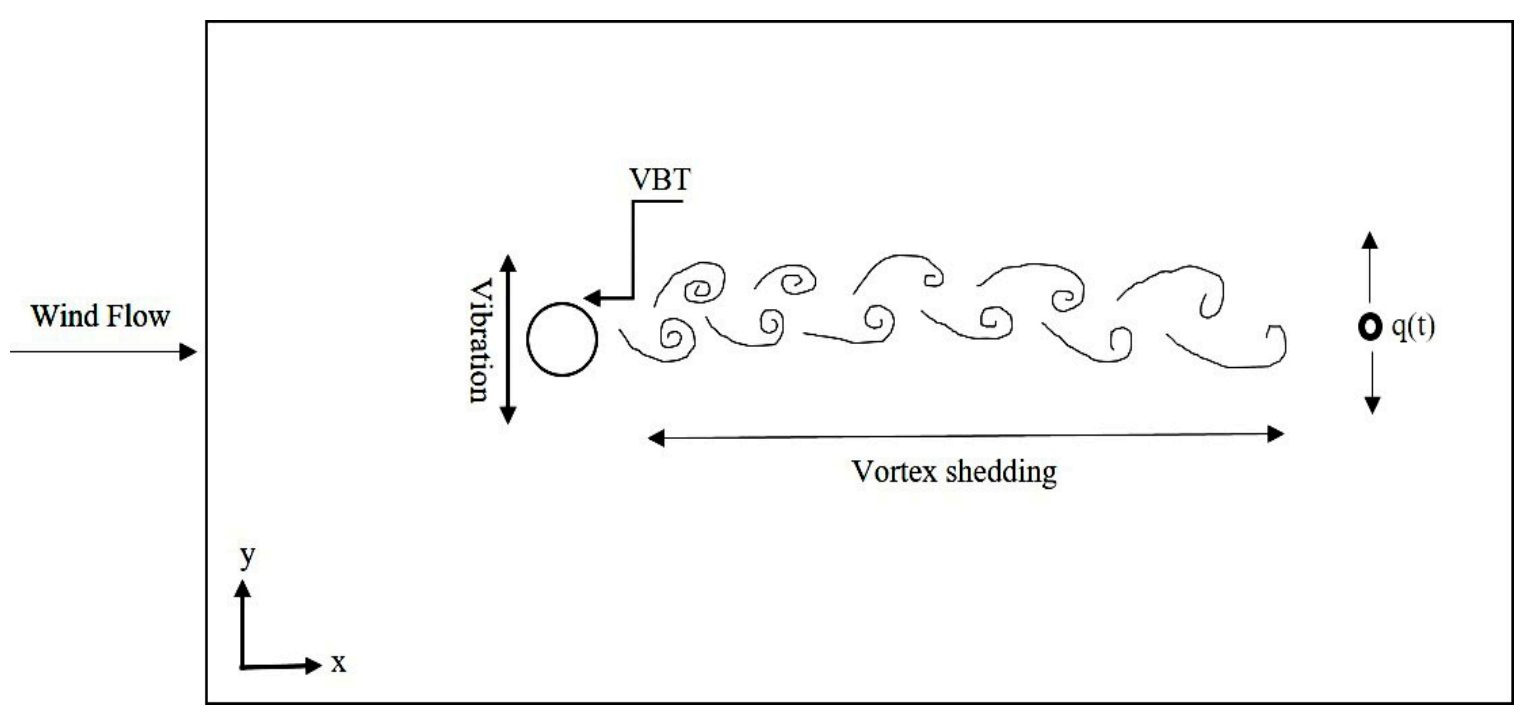

Figure 4. The schematic of the two-way effect of vortex-induced vibration.

The vortex shedding caused by vibration is a phenomenon that occurs when fluid interacts with the vibrational structure, and it is a function of the Reynolds number. The coupled two equations of the VIV phenomenon are describing the effect of wind flow on the VBT structures, and vice versa, are as follows, according to [30].

$$
\begin{gathered}
m \ddot{y}+k y+c \dot{y}=\frac{1}{2} \rho u^{2}(D l) C_{d}(x, y) \sin (\omega t+\varphi) \hat{i}+\frac{1}{2} \rho v^{2}(D l) C_{d}(x, y) \sin (\omega t+\varphi) \hat{j} m \ddot{y}+k y+c \dot{y} \\
\ddot{q}+\varepsilon \omega_{f}\left(q^{2}-1\right) \dot{q}+\omega_{f}^{2} q=A \ddot{y} \ddot{q}+\varepsilon \omega_{f}\left(q^{2}-1\right) \dot{q}+\omega_{f}^{2} q=A \ddot{y}
\end{gathered}
$$

where $\omega_{f}$ is the vorticity angular velocity, and $q$ is the strength of the vortices behind VBT. It should be noticed that the VIV phenomenon depends on some different structural and fluid parameters, such as the flow velocity to the structure stiffness. Hence, replacing the 
experimental value of some parameters can help to simplify the procedure of these coupled equations [31]. The system of non-linear and differential coupled equations of solid and fluid was solved by the fourth-order Runge-Kutta method by commercial software.

\subsection{Energy Harvesting}

One of the main parts of this research is a numerical analysis of harvesting electrical energy from the VBT vibration while wind flows in a domain. To achieve this purpose, the Faraday law of induction is used in order to help determine the electrical energy harvested from the VBT vibration. The mechanical power absorbed by the VBT $\left(P_{\text {wind }}\right)$ and the produced electrical power $\left(P_{V B T}\right)$ are as follows, respectively [32]:

$$
\begin{gathered}
P_{V B T}=\eta P_{\text {wind }} \\
P_{V B T}=\eta \frac{1}{2} \rho u^{3}(2 y+D) l
\end{gathered}
$$

\subsection{Machine Learning Method}

One of the essential goals in the AI method is to design an algorithm for building a relationship between input and output data. By utilizing the numerical analysis, the dataset was imported to the AI algorithm to predict the output parameters.

The selected algorithm for the recent study was long short-term memory (LSTM). LSTM is one of the Deep Neural Network (DNN) methods applicable for detecting a different type of time series data [33]. It fundamentally uses a multilayer neural network to learn a time-series relationship between the input and output parameters [34]. The data accumulated from the sensors in experiments and simulations are time-dependent, so LSTM is the best algorithm for predicting output data [35]. Hence, this algorithm has a feedback connection, unlike other neural networks. In other words, LSTM is practical for the applied architecture in long-term. Furthermore, to store the data information that is used in the long-term storage in hidden layers, the "cell-states" were introduced. As presented in Equations (10) and (11), $f_{t}$ and $i_{t}$ introduce the forget and input gates for controlling the input and output of each cell-state [36].

$$
\begin{gathered}
f_{t}=g\left(W_{f} \cdot\left[h_{t-1} \cdot X_{t}\right]+b_{f}\right) \\
i_{t}=g\left(W_{i} \cdot\left[h_{t-1} \cdot X_{t}\right]+b_{i}\right)
\end{gathered}
$$

where the function $g$ introduces a non-linear sigmoid function, which is used during the activating procedure. $\mathrm{f}$ and $\mathrm{i}$ indexes show the forget and input parameters, $W$ and $b$ introduce the weight matrix and bias function, $h_{t-1}$ shows the output vector of the last time step, and $X_{t}$ presents the input vector of the current time step. Furthermore, to gain the input of the current state, Equation (12) presents the relation.

$$
C_{t}^{\prime}=\tanh \left(W_{c} \cdot\left[h_{t-1} \cdot X_{t}\right]+b_{c}\right)
$$

In this equation, the $c$ index shows the current state of each parameter. Equation (13) obtains the current cell state, which is considered as using both forget and input gates [36].

$$
C_{t}=f_{t} * C_{t-1}+i_{t} \times C_{t}{ }^{\prime}
$$

By using the output gate of each cell-state, as shown in Equation (13), the output of long short-term memory is presented as Equation (14).

$$
O_{t}=g\left(W_{o} \cdot\left[h_{t-1} \cdot X_{t}\right]+b_{o}\right)
$$

where the $o$ index shows the cell-state output parameters.

$$
h_{t}=O_{t} * \tanh \left(C_{t}\right)
$$


Figure 5 shows the cell-state of the LSTM method in predicting the produced power of VBT.

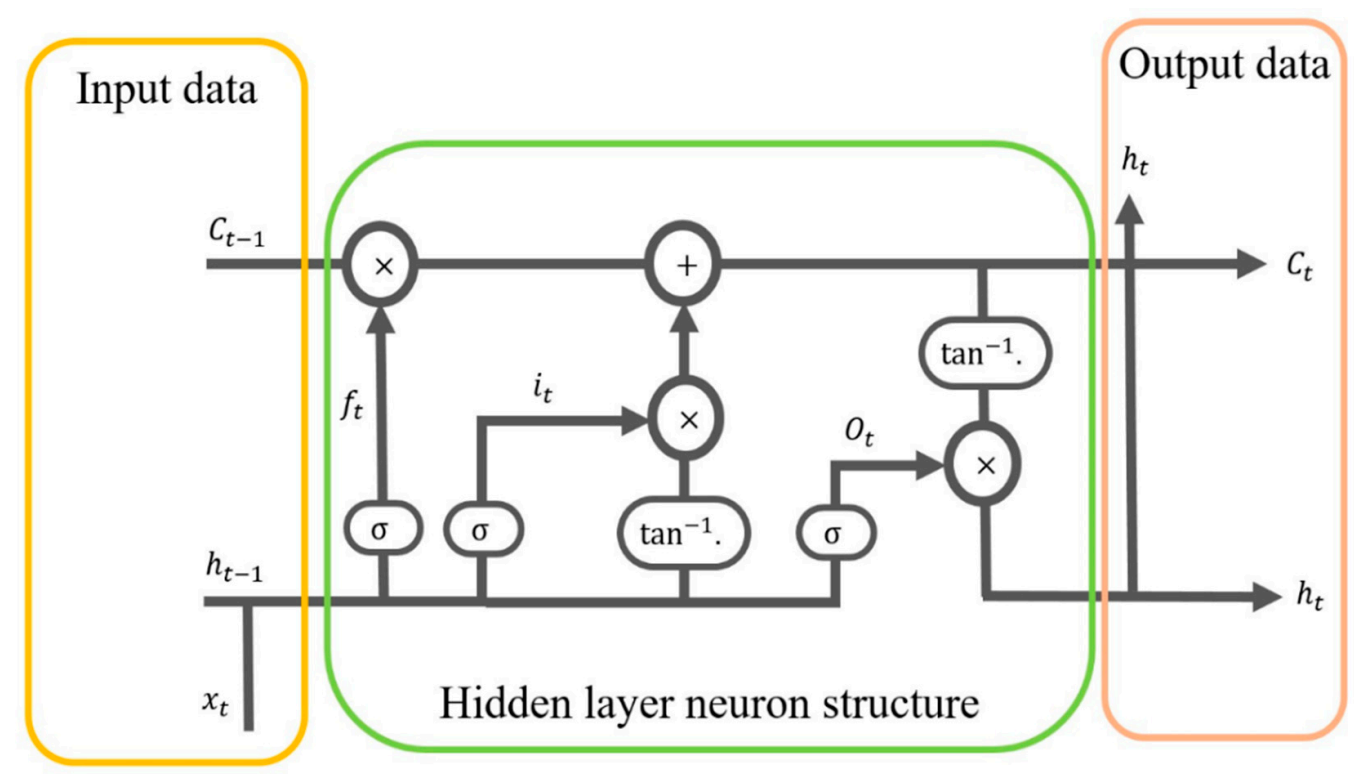

Figure 5. The long short-term memory diagram of a cell-state.

In this figure, different operators are indicated as numbers 1-7. Each number demonstrates the different state of data, which is shown in Table 2.

Table 2. The definition of different signs of the long short-term memory diagram of a cell-state.

\begin{tabular}{cc}
\hline Number & Definition \\
\hline 1 & forget some cell content \\
\hline 2 & compute the forget gate \\
\hline 3 & compute the input gate \\
\hline 4 & compute the new cell content \\
\hline 5 & compute the output gate \\
\hline 6 & write some new cell content \\
\hline 7 &
\end{tabular}

Different training steps based on an LSTM neural network according to [37] are described as follows. 1 . The $t-1$ time data feature is input to the input layer, then the output goes to the main cell of time, and finally, after calculations, the output of the t-time cell goes into the last cell, which is called the $t+1$ time cell. 2 . The output data of the hidden neuron layer comes from the input, forget, and output gates of each cell. 3 . The output results are formed by selecting between output LSTM nodes in the last neuron layer. 4. The error is backpropagated during the updating procedure of weight functions.

In this research, 5 hidden layers were selected, containing 10 neurons within the first layer. The epoch size is 200 , and the walk-forward validation method was used. The backpropagation algorithm was utilized for the administered learning technique. Hence, the quantity reduction procedure of the input data was performed by the Mahalanobis distance (MD) method to reduce the training and prediction time of the whole network [38]. Then, the data collected from numerical analysis would be compared with the predicted data from the LSTM method. Finally, the residual signal is applied on a detection step of the occurrence of faults. 


\section{Results and Discussion}

In the present research, the numerical solutions were performed to collect data for use in the LSTM algorithm. The selected dataset contains the data of $200 \mathrm{~s}$ of VBT simulation inflow. However, the electrical output power was calculated by utilizing a relation of the generator output power. The datasets have fewer data stores than expected in the case of real industrial problems.

Due to the comparability of this study, it was preferred for the simulation results and the LSTM predictions to be present in the same figure for each studied parameter. In order to find the relationship between the parameter and the variables used in this research, it was necessary to use a correlation matrix. In this matrix, each row and column represents a parameter, and each element of the matrix represents a graph that shows the relationship between them, as shown in Figure 6. Testing time, wind flow speed $(\mathrm{m} / \mathrm{s})$, drag force $(\mathrm{N})$, and the VBT vibration amplitude $(\mathrm{m})$ are the parameters analyzed in a correlation matrix and expressed as a value between 0 to 1 .

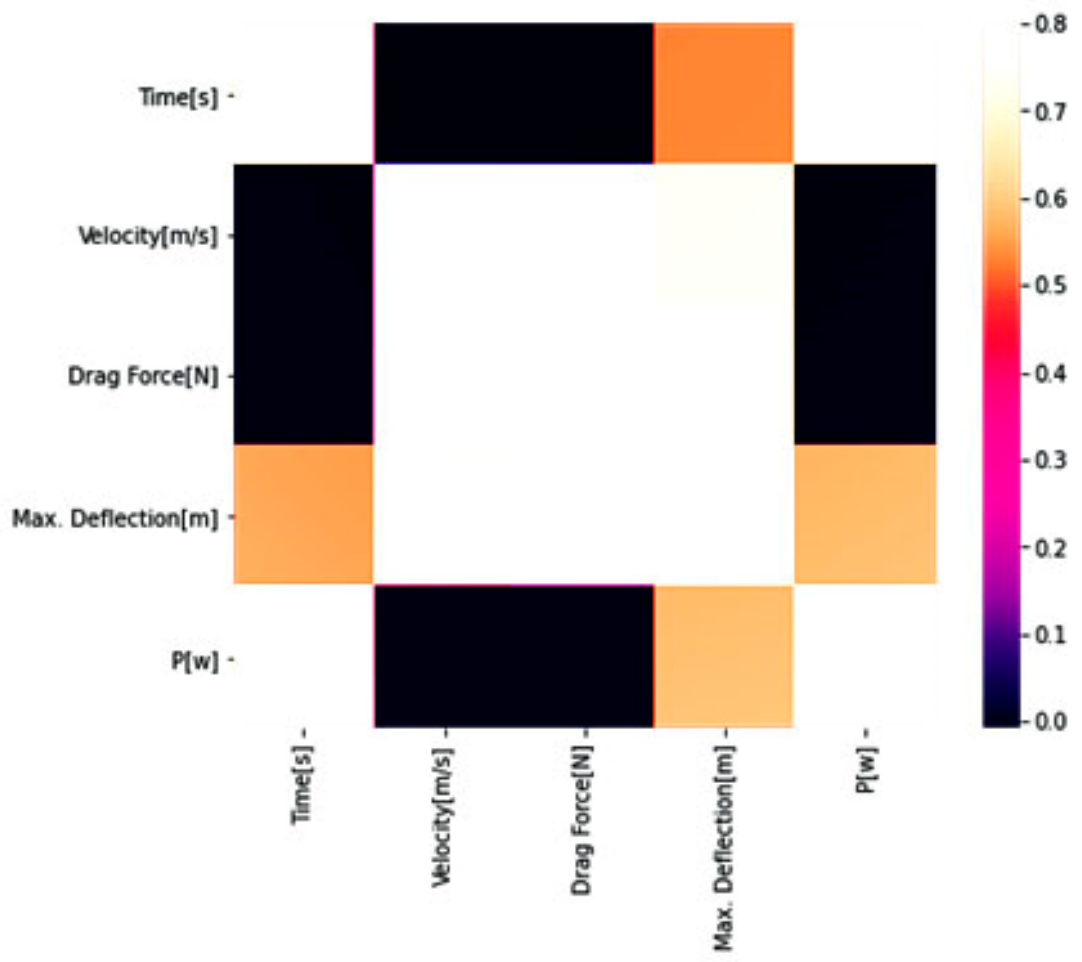

Figure 6. The correlation matrix of different parameters.

As shown in Figure 6, this matrix shows the relation of different quantities with a heat-map visualization. It demonstrates the magnitudes with colors from light to dark. The lighter color shows the best relation so it is easy to infer that the prediction procedure finds a good relation between time and output power. As presented in Figure 6, the best correlation is related to the maximum rod deflection and wind velocity, maximum rod deflection, and drag force, and wind velocity and drag force. These correlations have the least RMSE values.

The results of the data prediction are presented by graphs. Figure 7 shows the scatter plot of predicted magnitudes. It should be noticed that the positive magnitudes of wind flow speed and drag force have been evaluated by numerical solutions but the AI method has predicted both negative and positive values. Furthermore, Figure 8 shows the linear regression of the correlation scatter data. This figure is the same as Figure 8, but the points are connected with the best fitting line. 

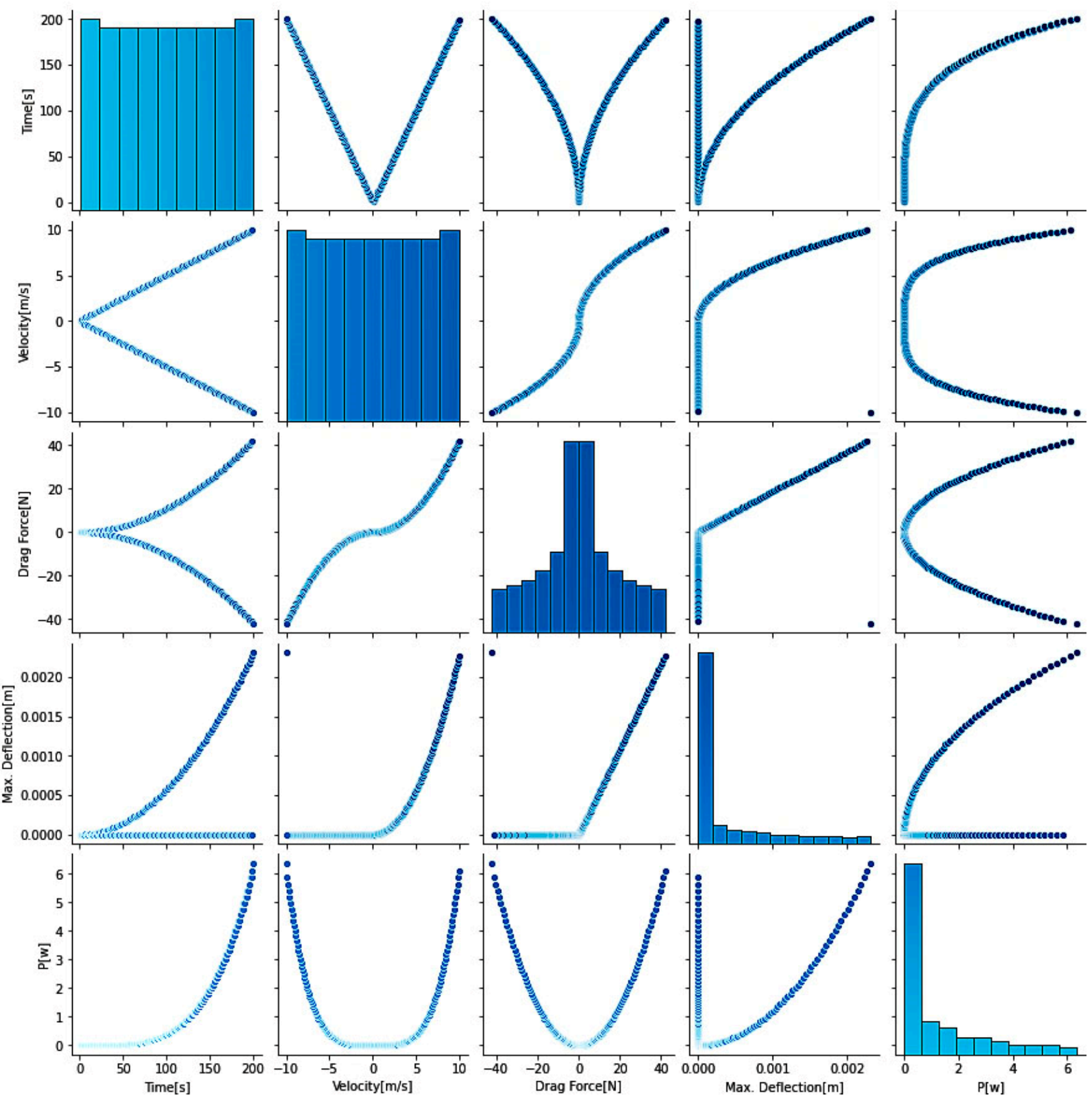

Figure 7. The overall figures of different parameter correlations.

Figure 7 is the optimization result of Figure 8, which presents a gradient descent of different variables by optimizing the cost function. As presented in these two figures, by increasing the wind speed over time, the rod deflection will be increased, and it causes the increasing trend in drag force. According to the energy conservation law, an slight rise in mechanical energy values would lead to the increasing trends in electrical energy. Consequently, the output power will increase. These charts show how the answers are optimized to find the best fit. To evaluate the effectiveness of the prediction method, the numerical analysis data, which are collected from the FSI simulation of VBT, have been compared with the LSTM method results. The Mahalanobis distance parameter is a combination of produced electrical power, wind flow velocity, amplitude, and drag force as an algorithm input dataset. The statistical details of count, mean, and standard deviation of the generated power are shown in Table 3. The mean and median magnitude of generated electrical power is 1.2 and $0.38 \mathrm{w}$, which is related to the information about VBT [33]. 

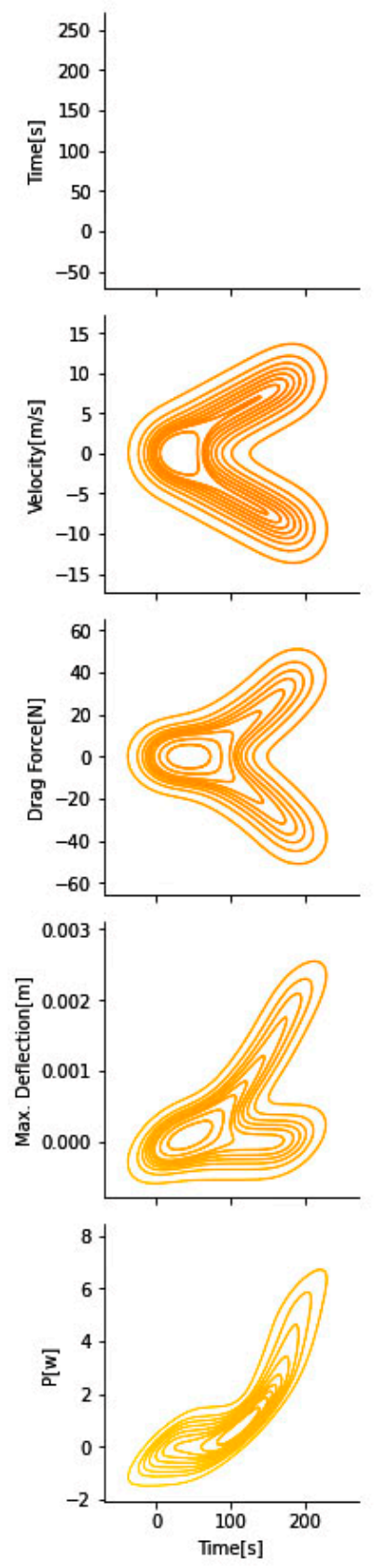
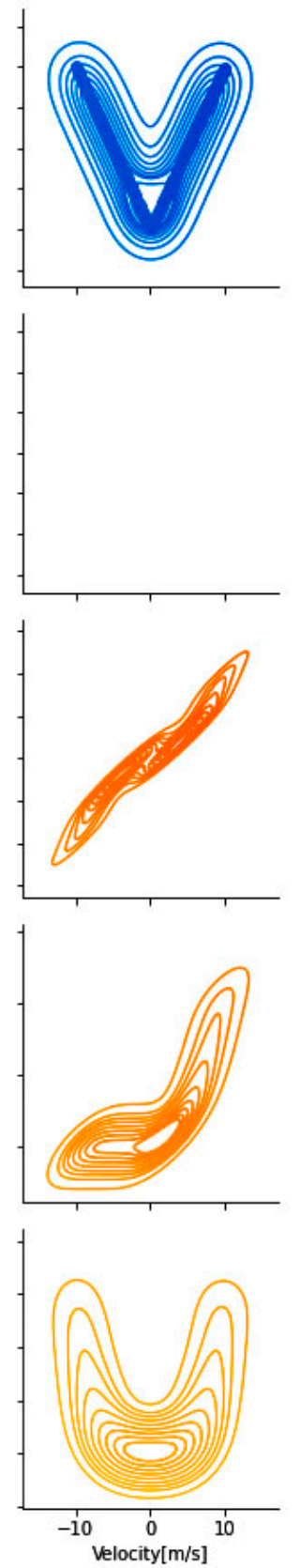
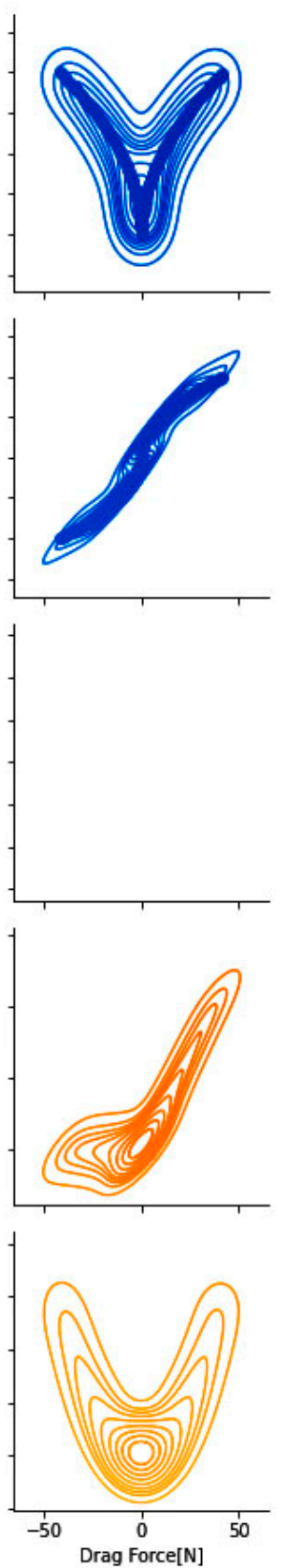
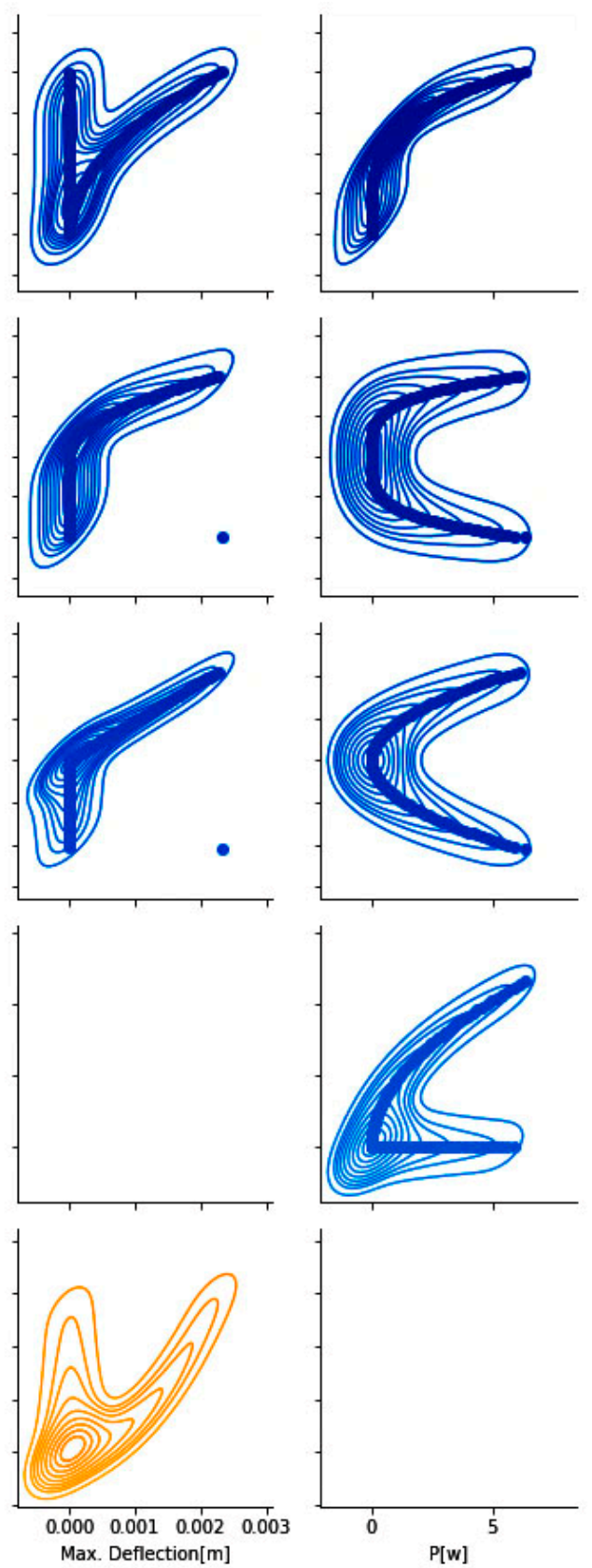

Figure 8. The overall figures of different parameter correlations.

Table 3. Statistical descriptions of the numerical solution.

\begin{tabular}{cc}
\hline Name & Power (kw) \\
\hline Count & 200 \\
\hline Mean & 1.242593 \\
\hline Standard Deviation & 1.669378 \\
\hline Minimum & 0.00 \\
\hline $25 \%$ & 0.023927 \\
\hline Median (50\%) & 0.382359 \\
\hline $75 \%$ & 1.934792 \\
\hline Maximum & 6.364972 \\
\hline
\end{tabular}


Figure 9 describes the output electrical power curve from VBT. It shows that the power is the third-order function of the input wind flow velocity in the range of 0 to $10 \mathrm{~m} / \mathrm{s}$. Hence, producing the electrical power in this special type of wind turbine starts from a low value of velocity. It should be noticed that the accuracy of prediction modeling is not only investigated by modeling parameters but also by the method of selected input variables, which is an important issue. In other words, another important factor that can impress the produced power is an oscillating amplitude. In this figure, the validation was performed, and it shows a good agreement. Furthermore, the respective equation is shown on the curve.

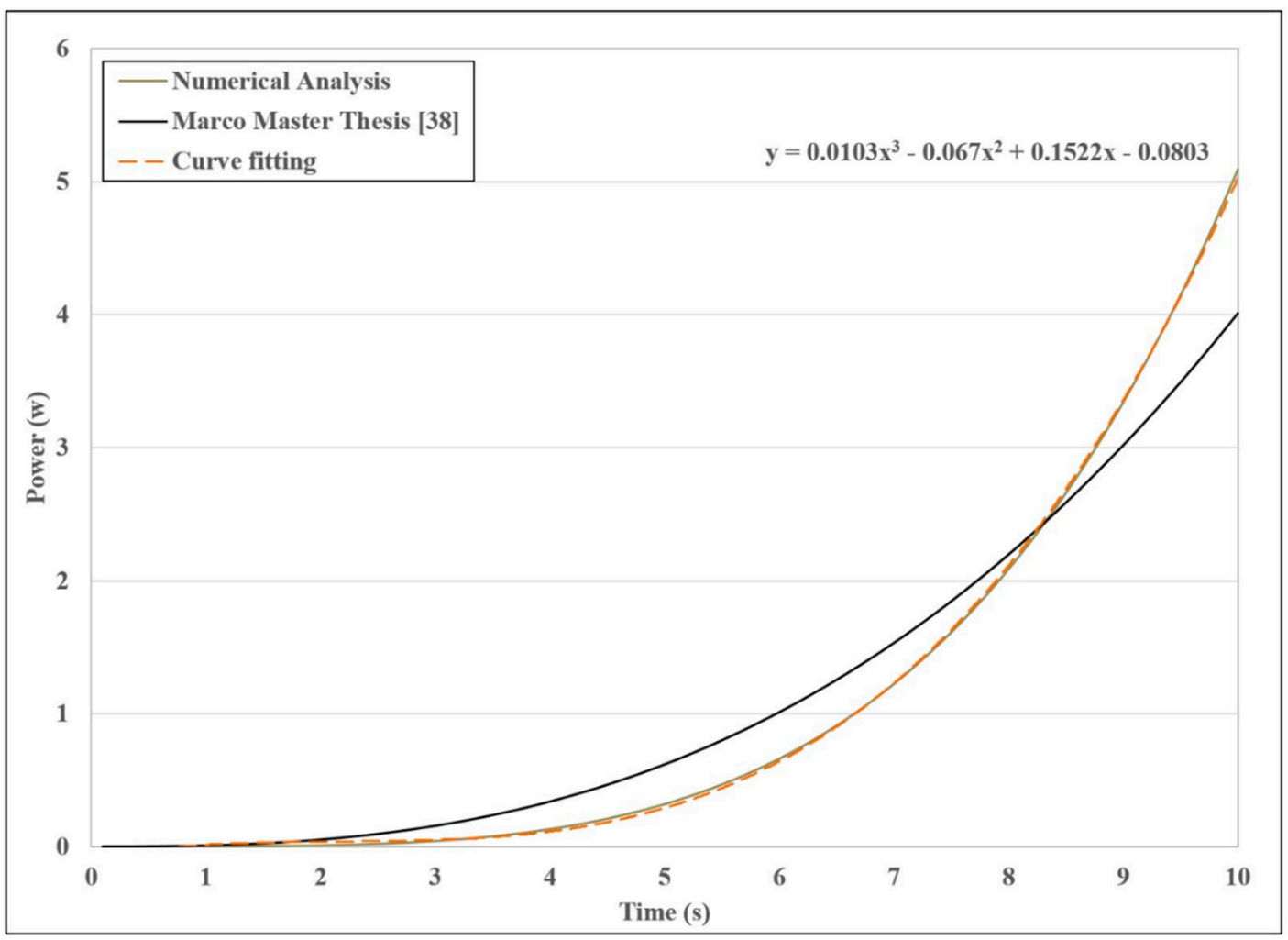

Figure 9. The effect of increasing the wind flow velocity on produced power.

As shown in Figure 9, the produced power is increasing with time. By comparing with another study [38], it has a low difference, which is related to some differences in the design and some VBT properties. Suggesting a mathematical relation between VBT output power and time is one of the novelties of recent research, which can be introduced as Equation (16). This equation consists of 180 generated data curve fittings during the LSTM analysis.

$$
P=0.0103 t^{3}-0.067 t^{2}+0.1522 t-0.0803
$$

Figure 10 presents the effect of changing the amplitude of vibration on electrical output power. It shows that the generated power has the same magnitude for both negative and positive values of VBT deflection.

As shown in Figure 10, by increasing the vibration amplitude, the produced power will be increased too. It is not important whether the amplitude is negative or positive as, in Faraday's law of induction, only deflection and movement are important to produce electricity. In this case, Figures 11-13 present the effect of the named parameters on each other. 


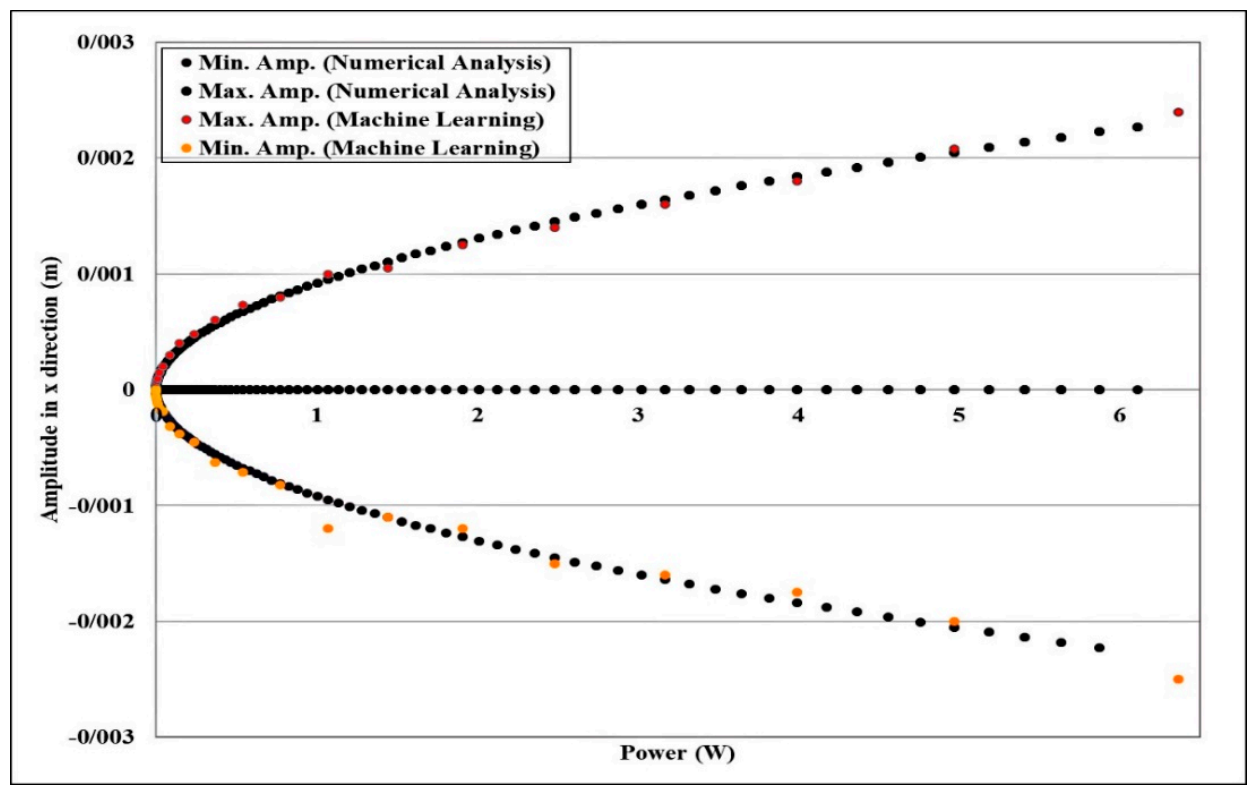

Figure 10. The effect of increasing the produced power on the amplitude of vibration.

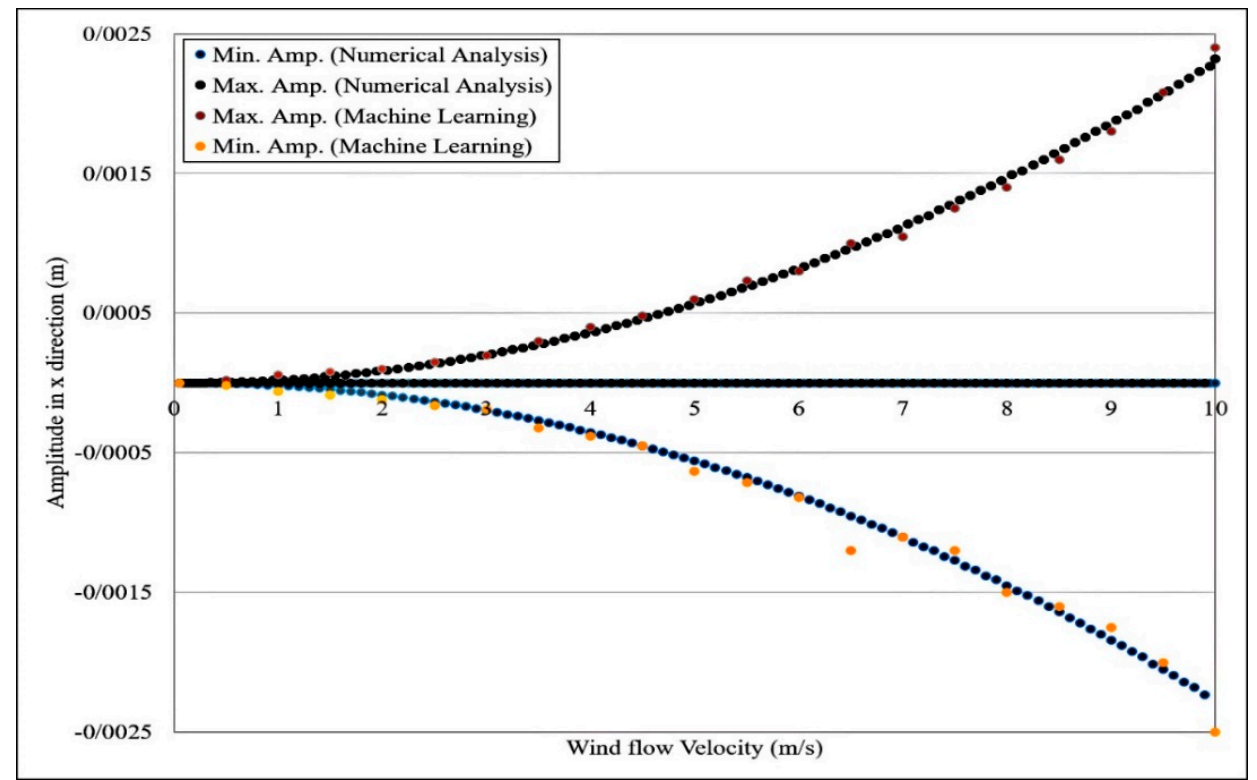

Figure 11. The effect of increasing the wind flow velocity on the amplitude of vibration.

As shown in Figure 11, the amplitude of the vibration, which is caused by the rod deflection, increases by increasing the wind flow velocity. The analysis demonstrated that all of the parameters change before the lock-in range $(10 \mathrm{~m} / \mathrm{s})$. As expected, by considering the other studies [36-38], the amplitude of vibration increases to gain $0.0023 \mathrm{~m}$ at the tip of the VBT. By having the comparative analysis of this figure, the predicted values of vibration amplitude are very close to the numerical analysis, and it proves that the LSTM method is accurate at predicting problems. 


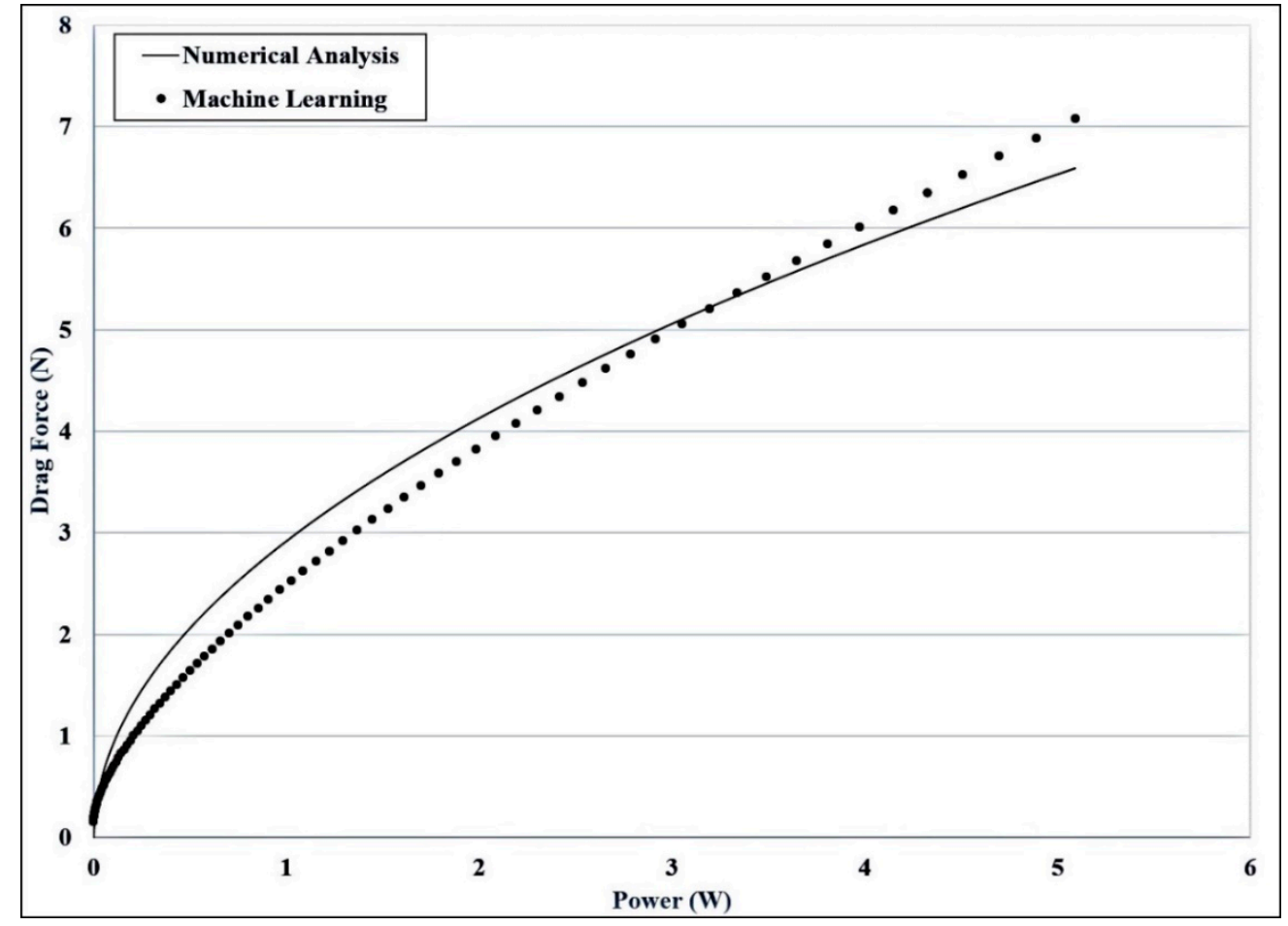

Figure 12. The effect of increasing the produced power on drag force.

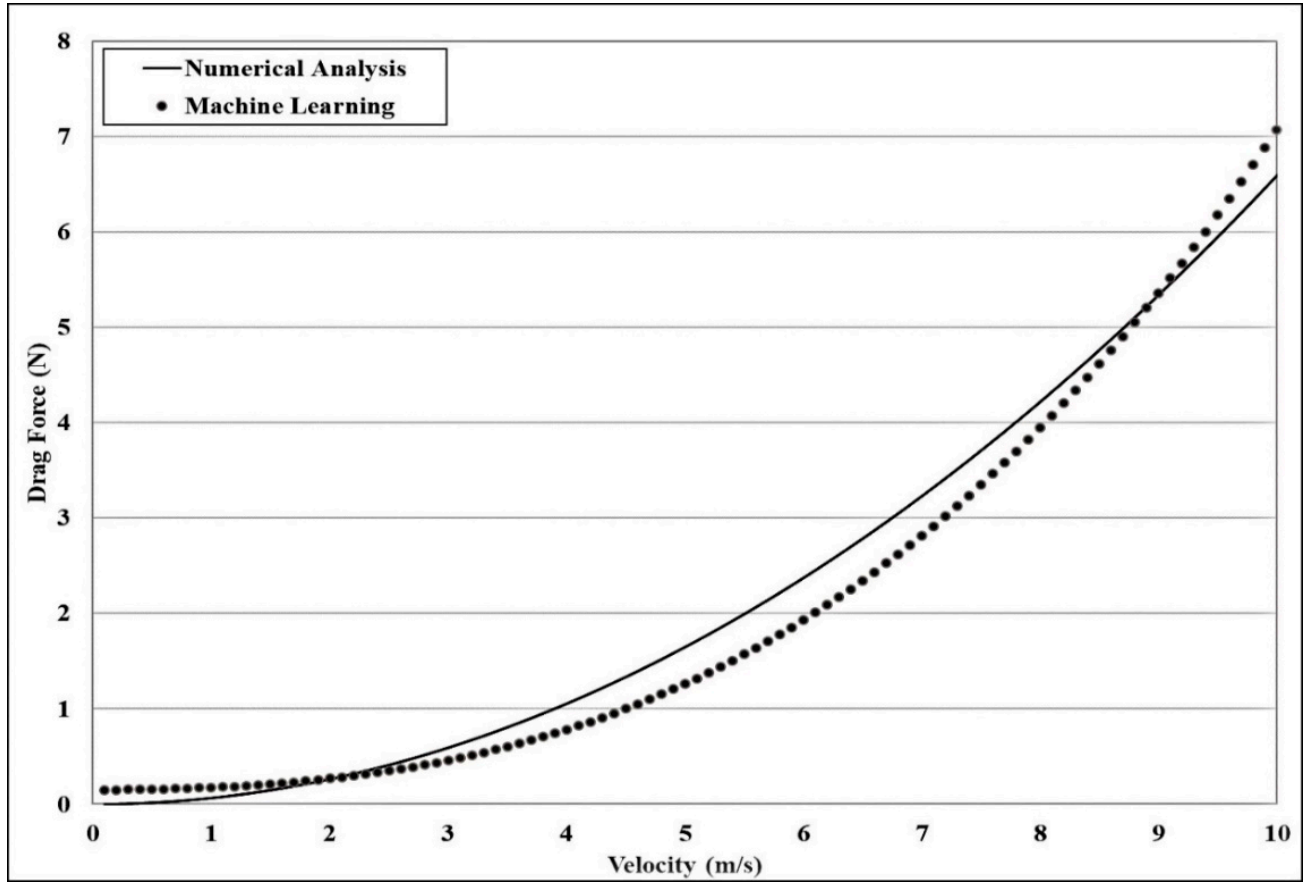

Figure 13. The effect of increasing the wind flow velocity on drag force.

As demonstrated in Figure 12, power is the second-order function of the drag force. As presented in this figure, by increasing the drag force, the produced electrical power will be increased to 6 watts. The maximum exerted drag force is nearly $6.5 \mathrm{~N}$ for the maximum value of power. This figure completely compares two different methods. The differences between the two named methods are small so we can conclude that AI is a reliable method in the field of detection. 
The effect of the wind flow velocity on the exerted drag force is studied in Figure 13. These values obtained from LSTM methods, presented in discussed figures, were compared with the results of the numerical investigations carried out by the CFD-FEM method. According to the comparative analyses performed for the important parameters in this research, it is possible to understand the performance of the artificial intelligence method by using the concept of root mean square error (RMSE) [22]. Five comparative relationships are explained here, as shown in Table 4.

Table 4. The accuracy of the LSTM model.

\begin{tabular}{cc}
\hline Input Variables & RSME Value \\
\hline Amplitude in x direction, Power Output & 0.18 \\
\hline Amplitude in x direction, Wind Speed & 0.20 \\
\hline Drag Force, Power Output & 0.43 \\
\hline Drag Force, Wind Flow Velocity & 0.41 \\
\hline $\begin{array}{c}\text { Wind Flow Velocity, Power Output, Drag Force } \\
\text { and Amplitude in x direction }\end{array}$ & 0.305 \\
\hline
\end{tabular}

Table 4 proves that there is a good agreement between numerical and artificial intelligence methods, and it can be concluded that the artificial intelligence method is very accurate and fast.

\section{Conclusions}

This paper contains a study in the field of produced power of the last generation of a wind turbine and a novel AI method to predict this parameter. In this paper, a novel machine learning prediction model of the VBT power based on LSTM has been investigated. One of the crucial problems that are solved in this research is predicting the produced power of VBT without spending any money. Related to the local wind speed, we can easily forecast how much power will be produced yearly. The dataset has been collected from a numerical analysis, which was performed by CFD with the finite element method in a field of FSI. The ideal number of grids was chosen for the purpose of the simulation, and the calculated generated power was validated with another study. Hence, different effective parameters that can affect the produced electrical power of VBT have been investigated. The variation of produced electrical power of VBT was studied with changeable parameters through two different solution methods. It has been proved that the vibration amplitude increased with the increasing wind flow velocity up to the received wind velocity of $10 \mathrm{~m} / \mathrm{s}$, which is a lock-in phenomenon. This particular point is where the maximum power output produced by this type of turbine occurs. For this reason, engineers and researchers in this field are trying to design this type of turbine so that the maximum vibrations of the VBT structure occur in this particular interval. In this study, the lock-in range occurs at a speed of $10 \mathrm{~m}$ per second, and the amplitude of the oscillation at this speed is about $0.0025 \mathrm{~m}$. According to these concepts and studies, the power at this particular point is about 5 watts.

In this study, two different methods were compared, and the main goal of this research is to have a comparative analytical solution for simulating the VBT through predicting the power. The prediction procedure was performed by using different effective parameters based on the derived LSTM algorithm. The correlation matrix presents the relation of different parameters. By utilizing the RMSE value, it has been shown that they were in good agreement with the results of the numerical analysis. For comparative analysis between the two methods, RSME values of the parameters were calculated, and the mean value obtained was 0.3 . This value proves the very high efficiency of this method in predicting the desired values. The proposed method had shown to be computationally fast and cheap, and it is expected to outperform the numerical methods. Further research however, would be essential to compare the model accuracies. 
Author Contributions: M.D.M. and M.G.—conceptualization; M.D.M. and S.M.M.-simulation; M.D.M., S.M.M. and A.H.M.—methodology; M.D.M. and A.H.M.—software; M.G. and L.K.supervision; M.G., A.H.M. and L.K.—writing and review; M.D.M., M.G., S.M.M., A.H.M. and L.K.-administration; A.H.M.-funding acquisition. All authors have read and agreed to the published version of the manuscript.

Funding: Project no. 2019-1.3.1-KK-2019-00007 has been implemented with the support provided by the National Research, Development and Innovation Fund of Hungary, financed under the 2019-1.2.1-KK funding scheme.

Institutional Review Board Statement: Not applicable.

Informed Consent Statement: Not applicable.

Data Availability Statement: Not applicable.

Acknowledgments: L.K. was supported by the Eötvös Lóránd Research Network Secretariat under grant agreement no. ELKH KÖ-40-2020 (development of cyber-medical systems based on AI and hybrid cloud methods).

Conflicts of Interest: The authors declare that they have no known competing financial interests or personal relationships that could have appeared to influence the work reported in this paper.

\section{References}

1. Manisalidis, I.; Stavropoulou, E.; Stavropoulos, A.; Bezirtzoglou, E. Environmental and health impacts of air pollution: A review. Front. Public Health 2020, 8. [CrossRef]

2. Berk, I.; Kasman, A.; Kılınç, D. Towards a common renewable future: The System-GMM approach to assess the convergence in renewable energy consumption of EU countries. Energy Econ. 2020, 87, 103922. [CrossRef]

3. Martin, S.; Jung, S.; Vanli, A. Impact of near-future turbine technology on the wind power potential of low wind regions. Appl. Energy. 2020, 272, 115251. [CrossRef]

4. Jahangir, M.H.; Shahsavari, A.; Rad, M.A. Feasibility study of a zero emission PV/Wind turbine/Wave energy converter hybrid system for stand-alone power supply: A case study. J. Clean. Prod. 2020, 262, 121250. [CrossRef]

5. Mostafaeipour, A.; Rezaei, M.; Jahangiri, M.; Qolipour, M. Feasibility analysis of a new tree-shaped wind turbine for urban application: A case study. Energy Environ. 2020, 31, 1230-1256. [CrossRef]

6. Salvador, C.S.; Teresa, J.A.; Martinez, J.M.; Cavin Bacasnot, M.; Vincent Orilla, K.; Joshua Cabana, R.; Iza Ladaran, W. (Eds.) Design and Construction of Arc Shaped and Disc Shaped Pendulum for Vortex Bladeless Wind Generator. In Proceedings of the 25th International Conference on Systems Engineering (ICSEng), Las Vegas, NV, USA, 22-24 August 2017; IEEE: Piscataway, NJ, USA, 2017. [CrossRef]

7. Available online: https://vortexbladeless.com/technology-design/ (accessed on 4 April 2021).

8. Anthony Adeyanju, A.; Boucher, D. Theoretical Analysis of the Bladeless Wind Turbine Performance. J. Sci. Res. Reports 2020, 26, 93-106. [CrossRef]

9. Sassi, P.; Freiría, J.; Mendina, M.; Draper, M.; Usera, G. Simulation of vorticity wind turbines. Heliyon 2020, 22, 6-26. [CrossRef] [PubMed]

10. Gautam, A.; Srinivas, S.; Ravi Teja, A. Efficient Electro-Mechanical Conversion System in Bladeless Wind Turbines. In Proceedings of the 2020 IEEE 29th International Symposium on Industrial Electronics (ISIE), Delft, The Netherlands, 17-19 June 2020; Volume 29, pp. 1009-1014. [CrossRef]

11. Moradi Gharghani, F.; Bijarchi, M.; Mohammadi, O.; Shafii, M.B. An experimental investigation into a novelsmall-scale device for energy harvestingusing vortex-induced vibration. Int. J. Low-Carbon Technol. 2020, 1-9. [CrossRef]

12. Gibadullin, A.A.; Sadriddinov, M.I.; Kurbonova, Z.M.; Shedko, Y.N.; Shamraeva, V.V. Assessment of Factors Ensuring Sustainable Development of the Electric Power Industry in the Context of Transition to Renewable Energy Sources of the National Economy. In IOP Conference Series: Earth and Environmental Science; IOP Publishing: Moscow, Russia, 2020; Volume 421, p. 032051. [CrossRef]

13. Mbungu, N.T.; Naidoo, R.M.; Bansal, R.C.; Siti, M.W.; Tungadio, D.H. An overview of renewable energy resources and grid integration for commercial building applications. J. Energy Storage 2020, 29, 101385. [CrossRef]

14. Zhou, B.; Duan, H.; Wu, Q.; Wang, H.; Or, S.W.; Chan, K.W.; Meng, Y. Short-term prediction of wind power and its ramp events based on semisupervised generative adversarial network. Electr. Power Energy Syst. 2021, 125, 106411. [CrossRef]

15. Gao, Y.; Wang, M.; Ji, R.; Wu, X.; Dai, Q. 3-D object retrieval with Hausdorff distance learning. IEEE Trans. Ind. Electron. 2013, 61, 2088-2098. [CrossRef]

16. Hanifi, S.; Liu, X.; Lin, Z.; Lotfian, S.A. A critical review of wind power forecasting methods-Past, present and future. Energies 2020, 13, 3764. [CrossRef]

17. Adedeji, P.A.; Akinlabi, S.; Madushele, N.; Olatunji, O.O. Wind turbine power output very short-term forecast: A comparative study of data clustering techniques in a PSO-ANFIS model. J. Clean. Prod. 2020, 254, 120135. [CrossRef] 
18. Yang, M.; Shi, C.; Liu, H. Day-ahead wind power forecasting based on the clustering of equivalent power curves. Energy $2021,218$. [CrossRef]

19. Wu, Q.; Guan, F.; Lv, C.; Huang, Y. Ultra-short-term multi-step wind power forecasting based on CNN-LSTM. IET Renew. Power Gener. 2021. [CrossRef]

20. Meka, R.; Alaeddini, A.; Bhaganagar, K.A. Robust deep learning framework for short-term wind power forecast of a full-scale wind farm using atmospheric variables. Energy 2021, 221, 119759. [CrossRef]

21. Cajas, J.C.; Houzeaux, G.; Yáñez, D. SHAPE project Vortex Bladeless: Parallel multi-code coupling for fluid-structure interaction in wind energy generation. Adv. Comput. Eur. 2016, 12, 1-6. Available online: http://hdl.handle.net/2117/88263 (accessed on 4 April 2021).

22. Moayedi, H.; Mosavi, A. An Innovative Metaheuristic Strategy for Solar Energy Management through a Neural Networks Framework. Energies 2021, 14, 1196. [CrossRef]

23. Thomai, M.P.; Kharsati, L.; Rama Samy, N.; Sivamani, S.; Venkatesan, H. Experimental Analysis of Vortex Induced Vibration in the Bladeless Small Wind Turbine. In Proceedings of the ASME 2019 Gas Turbine India Conference, Chennai, India, 5 December 2019; American Society of Mechanical Engineers Digital Collection: Shanghai, China. [CrossRef]

24. Xu, H. Application of intelligent control technology in aero-generator's overload protection. In Proceedings of the 2020 IEEE International Conference on Information Technology, Big Data and Artificial Intelligence (ICIBA), Chongqing, China, 6 November 2020; IEEE: Piscataway, NJ, USA, 2020; Volume 1, pp. 309-312. [CrossRef]

25. Available online: https://www.engineeringtoolbox.com/air-properties-d_156.html (accessed on 4 April 2021).

26. Singh, N. Simulation of flow over a rotationally oscillating square cylinder at low Reynolds numbers. Int. J. Automot. Mech. Eng. 2019, 16, 6368-6385. [CrossRef]

27. Hasheminejad, S.M.; Jarrahi, M. Numerical simulation of two dimensional vortex-induced vibrations of an elliptic 453 cylinder at low Reynolds numbers. Comput. Fluids 2015, 107, 25-42. [CrossRef]

28. Barrero-Gil, A.; Pindado, S.; Avila, S. Extracting energy from vortex-induced vibrations: A parametric study. Appl. Math. model. 2012, 36, 3153-3160. [CrossRef]

29. Wang, J.; Geng, L.; Ding, L.; Zhu, H.; Yurchenko, D. The state-of-the-art review on energy harvesting from flow-induced vibrations. Appl. Energy 2020, 267, 114902. [CrossRef]

30. Maftouni, N.; Dehghan Manshadi, M.; Mousavi, S.M. The Effect of Drag Force on The Body Frequencies and The Power Spectrum of a Bladeless Wind Turbine. Trans. Can. Soc. Mech. Eng. 2021. [CrossRef]

31. Yazdi, E.A. Nonlinear model predictive control of a vortex-induced vibrations bladeless wind turbine. Smart Mater. Struct. 2018, 27, 075005. [CrossRef]

32. Chang, Y.; Chen, J.; Qu, C.; Pan, T. Intelligent fault diagnosis of wind turbines via a deep learning network using parallel convolution layers with multi-scale kernels. Renew. Energy 2020, 153, 205-213. [CrossRef]

33. Kouadri, A.; Hajji, M.; Harkat, M.F.; Abodayeh, K.; Mansouri, M.; Nounou, H.; Nounou, M. Hidden Markov model based principal component analysis for intelligent fault diagnosis of wind energy converter systems. Renew. Energy 2020, 150, 598-606. [CrossRef]

34. Chen, W.; Qiu, Y.; Feng, Y.; Li, Y.; Kusiak, A. Diagnosis of wind turbine faults with transfer learning algorithms. Renew. Energy 2021, 163, 2053-2067. [CrossRef]

35. Boretto, M. Bladeless Wind Energy Conversion. Ph.D. Thesis, Politecnico di Torino, Rome, Italy, 2019.

36. Qian, P.; Tian, X.; Kanfoud, J.; Lee, J.L.Y.; Gan, T.-H. A Novel Condition Monitoring Method of Wind Turbines Based on Long Short-Term Memory Neural Network. Energies 2019, 12, 3411. [CrossRef]

37. Mousavi, S.M.; Ghasemi, M.; Dehghan Manshadi, M.; Mosavi, A. Deep Learning for Wave Energy Converter Modeling Using Long Short-Term Memory. Mathematics 2021, 9, 871. [CrossRef]

38. Károly, A.I.; Fullér, R.; Galambos, P. Unsupervised clustering for deep learning: A tutorial survey. Acta Polytech. Hung. 2018, 15, 29-53. 